Case Report

\title{
Nasal Adenocarcinoma in a Horse with Metastasis to Lung, Liver, and Bone and Review of Metastasis in Nine Horses with Sinonasal Tumors
}

\author{
Ashley Hanna, ${ }^{1}$ Susanne M. Stieger-Vanegas, ${ }^{1}$ Jerry R. Heidel, ${ }^{2}$ Melissa Esser, ${ }^{1}$ \\ John Schlipf, ${ }^{1}$ and Jacob Mecham ${ }^{1}$ \\ ${ }^{1}$ Department of Clinical Sciences, College of Veterinary Medicine, Oregon State University, Magruder Hall, Corvallis, OR 97331, USA \\ ${ }^{2}$ Department of Biomedical Sciences, College of Veterinary Medicine, Oregon State University, Magruder Hall, Corvallis, \\ OR 97331, USA
}

Correspondence should be addressed to Susanne M. Stieger-Vanegas; susanne.stieger@oregonstate.edu

Received 14 May 2015; Revised 22 June 2015; Accepted 24 June 2015

Academic Editor: Franco Mutinelli

Copyright (C) 2015 Ashley Hanna et al. This is an open access article distributed under the Creative Commons Attribution License, which permits unrestricted use, distribution, and reproduction in any medium, provided the original work is properly cited.

Sinonasal neoplasia metastasizing to distant organs is rare in horses. This case report describes the clinical and imaging findings of a horse with sinonasal neoplasia, which had metastasized to the lung, liver, and humerus. Additionally, the prevalence of sinonasal neoplasia and their incidence of distant metastasis among horses that presented to the Oregon State University Veterinary Teaching Hospital (OSU-VTH) were estimated. Of 5,558 equine patients who presented to the OSU-VTH in the last nine years, $1.4 \%$ were diagnosed with sinonasal disease and $10.3 \%$ of these cases had sinonasal neoplasia with only one having confirmed distant metastasis. This case was an eleven-year-old quarter horse which was evaluated due to a history of a right forelimb lameness of three weeks duration. Two and a half months later he presented again, this time for unilateral epistaxis and persistent right forelimb lameness. Radiography of the right elbow noted an increasingly irregular, periosteal response and osteolytic lesion of the right distal humeral condyle. At the time of the second presentation, nasosinal endoscopy identified a lobulated mass in the region of the ethmoid turbinates. Histopathology of this mass revealed an adenocarcinoma of nasal origin with metastasis to the lung, liver, and right humerus.

\section{Introduction}

Adenocarcinomas in the horse are uncommon but have been reported to occur in a variety of locations including lung, intestine, kidney, skin, lacrimal gland, testes, ovary, mammary tissue, sinuses, and nasal passages [1-7]. Adenocarcinomas of nasal and paranasal sinus origin are rare tumors affecting equids but can cause significant morbidity. More commonly the tumors originate in the maxillary area than in the nasal area [1]. They are often aggressive, locally invasive neoplasias that carry a poor prognosis [8]. These tumors have been reported to extend from the nasal cavity and paranasal sinuses through the cribriform plate into neural tissue and often tend to metastasize to regional lymph nodes [1,9]. Distant metastases of nasal adenocarcinomas to the lung have been described in two horses; however, this appears to be a rare occurrence. This is likely in part secondary to the low number of affected animals submitted for necropsy and in part due to the limitations of the current diagnostic techniques available evaluating the horses $[1,8]$. Though there has been no evidence of equine nasal adenocarcinoma metastasis to bone, there are reports of intestinal and renal adenocarcinomas metastasizing to the axial and appendicular skeleton $[2,7,10]$.

Neoplasia involving the appendicular skeleton in horses can cause severe lameness that is often attributed to bone pain, soft tissue pathology, or a nonpathologic fracture. Metastatic lesions as well as primary bone neoplasia can cause significant osseous destruction, which is caused by an increased osteoclastic activity secondary to a dysregulation of the normal bone homeostasis. The osseous destruction in combination with focal inflammation and increasing 


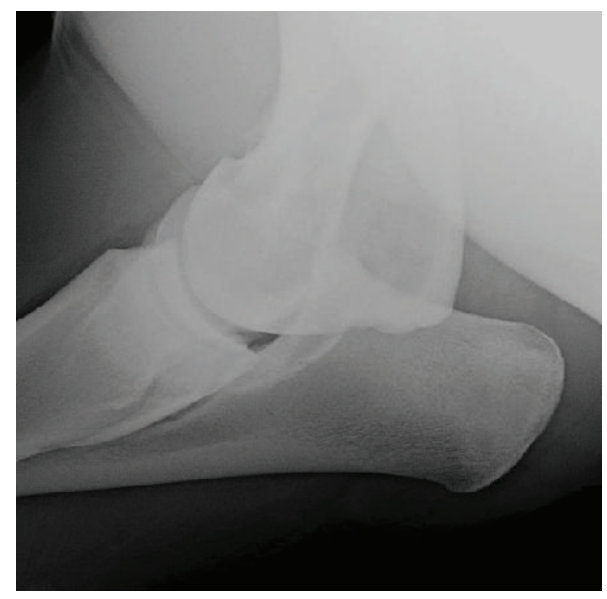

(a)

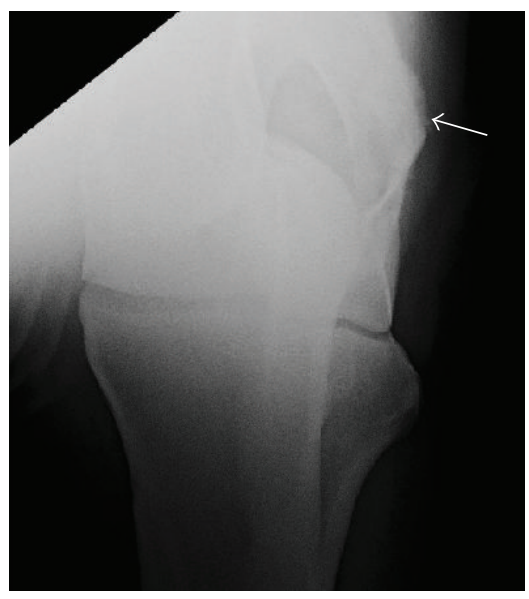

(b)

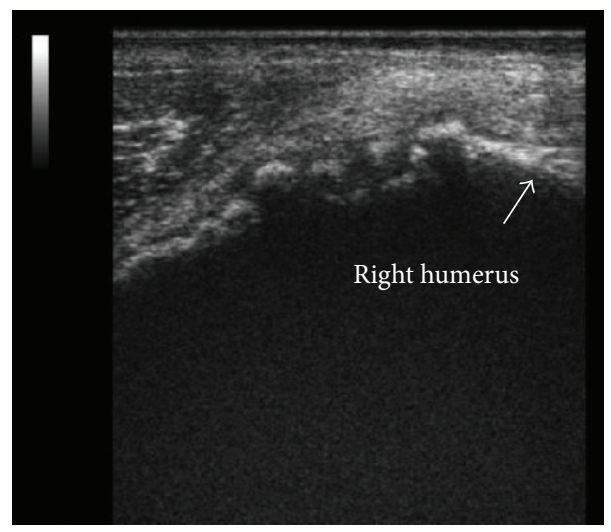

(c)

FIGURE 1: Radiographs of the right elbow at first presentation in (a) lateromedial and (b) craniocaudal views. A faint lucency is noted in the lateral aspect of the condyle of the right humerus. There is mild periosteal bone proliferation on the lateral aspect of the distal humerus metaphysis and condyle (marked by white arrow), which is best noted in the craniocaudal view. (c) Ultrasound image of the lateral aspect of the condyle of the right humerus. A marked irregularity of the bone surface just proximal of the origin of the lateral collateral ligament (marked by white arrow) of the right elbow joint is noted.

tissue acidity results in sensitized and partially destroyed peripheral nerve endings resulting in a high level of pain [11]. Osteosarcoma, fibrosarcoma, and chondrosarcoma involving bone have been documented infrequently in horses. In none of these horses metastatic lesions were present at necropsy, suggesting that the incidence of metastasis might also be low in these tumor types; however larger case series are needed to confirm this finding [12]. This case report describes a case of nasal adenocarcinoma with distant metastasis to multiple organs including liver, lung, and the distal right humerus. To the authors' knowledge this is the only report of nasal adenocarcinoma in the horse with metastasis to bone.

\section{Case Presentation}

An eleven-year-old quarter horse gelding used for pleasure riding first presented to the Oregon State University Veterinary Teaching Hospital (OSU-VTH) for a right forelimb lameness of three weeks' duration. He had been treated with oral flunixin meglumine (Banamine, Merck Animal Health, Whitehouse Station, NJ, USA) at a dose of $1.1 \mathrm{mg} / \mathrm{kg}$ once per day by the owners with no improvement. At presentation, the horse displayed a grade 4/5 right forelimb lameness, was dragging his toe, and was reluctant to further flexion of the limb. On physical examination, the horse stood with his right forelimb in slight flexion and his toe pointed. He had a positive response to hoof testers on his right forelimb. Palpation of the right forelimb elicited a mild pain response at the origin of the lateral collateral ligament of the elbow.

Localized abaxial sesamoid perineural anesthesia of the right forelimb did not improve the lameness. Intra-articular anesthesia of the elbow joint resulted in mild improvement but did not resolve the lameness. Radiographs of the right elbow (Figures $1(\mathrm{a})$ and $1(\mathrm{~b})$ ) revealed a mild irregularity of the lateral cortex of the metaphysis of the humerus and the epicondyle of the humerus at the origin of the lateral collateral ligament (Figure 1(b)). Ultrasound of the origin of the lateral collateral ligament demonstrated irregular bony margins at the lateral cortex of the distal humerus metaphysis extending to the proximal aspect of the attachment of the lateral collateral ligament (Figure 1(c)), which at the time was 
thought to be consistent with trauma secondary to injury of the lateral collateral ligament.

The horse was sent home with instructions for stall rest, administration of $2.2 \mathrm{mg} / \mathrm{kg}$ of oral phenylbutazone (Butapaste, Butler Schein Animal Health, Dublin, Ohio, USA) twice per day, and application of a $5^{\prime \prime}$ strip of topical $1 \%$ diclofenac sodium (Surpass, Boehringer Ingelheim Vetmedica, St. Joseph, MO, USA) over the right elbow joint twice per day. According to the owner the phenylbutazone was not providing adequate analgesia and was discontinued. Therefore oral gabapentin (Neurontin, Pfizer, New York, NY, USA) at a dose of $4.5 \mathrm{mg} / \mathrm{kg}$ twice per day and oral firocoxib (Equioxx, Merial, Duluth, GA, USA) at a dose of $0.1 \mathrm{mg} / \mathrm{kg}$ once per day were prescribed. While receiving gabapentin and firocoxib the horse became sound at the walk. It was recommended to reevaluate the horse in two weeks, or earlier if the horse was not improving with treatment. The owner did not schedule a visit and was contacted by phone two weeks following the initial examination and reported that the horse was still sound at walk. No other concerns about the horse were voiced at that time.

Two and a half months after his initial visit the horse presented again with a complaint of a mild intermittent left sided epistaxis over the last two months. The most recent episode of epistaxis was more severe and longer in duration than previously. At the time of presentation the horse was receiving gabapentin and firocoxib at the doses previously prescribed. On physical exam the horse had a constant drip of blood coming from his left nostril. Rectal temperature, heart rate, and respiratory rate were within normal limits. Blood work revealed a packed cell volume of $35 \%$ (reference range $32-53 \%$ ) and total protein of $8 \mathrm{~g} / \mathrm{dL}$ (reference range $5.7-7.9 \mathrm{~g} / \mathrm{dL})$.

The day following admittance, endoscopy revealed a lobulated mass in the region of the left ethmoid turbinates. A biopsy of the mass was obtained and submitted for histopathology.

Radiographs of the skull (Figures 2(a), 2(b), and 2(c)) and right elbow (Figures 3(a) and 3(b)) were subsequently performed. Radiographs of the skull revealed a poorly defined, irregularly outlined soft tissue opacity in the region of the left ethmoid turbinates (Figure 2(c)). Radiographs of the right elbow were compared to the previous radiographs and moderate, irregular periosteal proliferation at the distolateral aspect of the humerus in the location of the origin of the lateral collateral ligament of the elbow was noted (Figures 3(a) and $3(\mathrm{~b})$ ). The periosteal proliferation was more pronounced than on the previous radiographs (Figures 1(a) and 1(b)). In addition a heterogeneous lucency was present in the distomedial aspect of the humeral condyle that was not appreciated on the previous radiographs. Based on the most recent radiographs, a healing avulsion fracture was considered unlikely. At this time an underlying aggressive process was suspected with the most likely differential diagnosis being neoplasia, and less likely osteomyelitis.

Histologically, the ethmoidal mass consisted of neoplastic epithelial cells arranged in tubuloacinar, tubular, and solid patterns, all of which were transected by bands of fibrovascular tissue harboring large numbers of mixed mononuclear inflammatory cells (Figure 4). The neoplastic cells featured moderate anisocytosis and anisokaryosis, with frequent mitotic figures. Mucous-filled spaces and foci of necrosis accompanied by neutrophilic inflammation interrupted the neoplastic cell populations. The findings were consistent with a diagnosis of nasal adenocarcinoma.

Due to multiple lesions with poor prognoses, the owner elected humane euthanasia. The horse underwent a complete necropsy. The mass in the region of the left ethmoid turbinates measured $9 \times 5 \times 3 \mathrm{~cm}$. It was attached to the turbinates near their caudal extent but did not penetrate the cribriform plate (Figure 2(d)). Dissection of the condyle of the right humerus showed areas of variable malacia (Figure 3(c)). Gross pathology in the lungs included patchy atelectasis, pulmonary edema, and foci of pleural thickening. There were no gross lesions present in any other organs. Samples of all major organs as well as those having visible lesions were fixed in neutral buffered formalin for histologic examination.

Histologic features of the mass from the left ethmoid turbinates were similar to those found in the previous biopsy and remained consistent with nasal adenocarcinoma (Figure 4(a)). The liver and lung had intravascular accumulations of similar clustered neoplastic epithelial cells; in the lung these cells formed tubuloacinar patterns. There were similar accumulations of epithelial cells invading the bone of the right distal humerus (Figure 4(b)). Based upon these findings and the absence of other potential primary tumors, a final diagnosis of nasal adenocarcinoma with metastasis to liver, lung, and distal humerus was made.

To estimate the prevalence of sinonasal neoplasia, medical records of the Oregon State University Veterinary Teaching Hospital from July 2004 to April 2013 were reviewed for equids diagnosed with sinonasal disease. Of these equids diagnosed with sinonasal disease, all equids with confirmed sinonasal neoplasia were evaluated for presenting clinical signs, diagnostic tests performed, and imaging and histopathologic findings. Of 5,558 equine patients that presented to the Oregon State University Veterinary Teaching Hospital in the last nine years, 78 horses and one mule were diagnosed with sinonasal disease $(1.4 \%$ of the total number of horses seen). Of these, seven horses and one mule were diagnosed with a neoplasia originating in the nasal or paranasal sinus $(0.14 \%$ of the total number of horses seen). All of the cases with sinonasal neoplasia presented with nasal discharge. $75 \%$ had unilateral nasal discharge. One horse started with unilateral nasal discharge, which became later bilateral. Only two of the cases with sinonasal neoplasia had epistaxis. In these 8 cases the following other changes were noted: facial deformities $(n=2,25 \%)$, external lymphadenopathy $(n=2,25 \%)$, dyspnea $(n=2,25 \%)$, fever $(n=1,13 \%)$, ocular discharge $(n=1,13 \%)$, exophthalmos $(n=1,13 \%)$, and lameness $(n=1,13 \%)$. The following diagnostic tests were performed in the 8 cases of sinonasal neoplasia: skull radiographs $(n=6,75 \%)$, endoscopy $(n=7$, $88 \%)$, sinusoscopy $(n=2,25 \%)$, biopsies $(n=6,75 \%)$, and necropsy $(n=4,50 \%)$. Histologic diagnoses of these tumors were lymphoma $(n=2)$, adenocarcinoma $(n=2)$, and squamous cell carcinoma, osteoma, respiratory transitional 


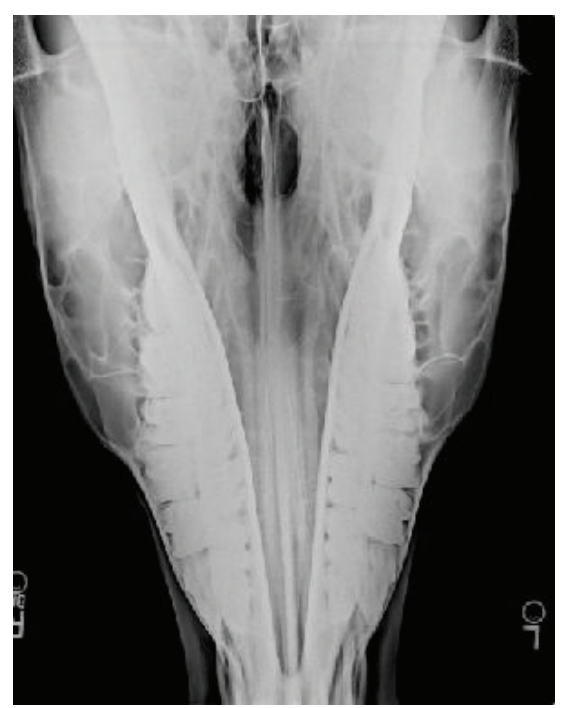

(a)

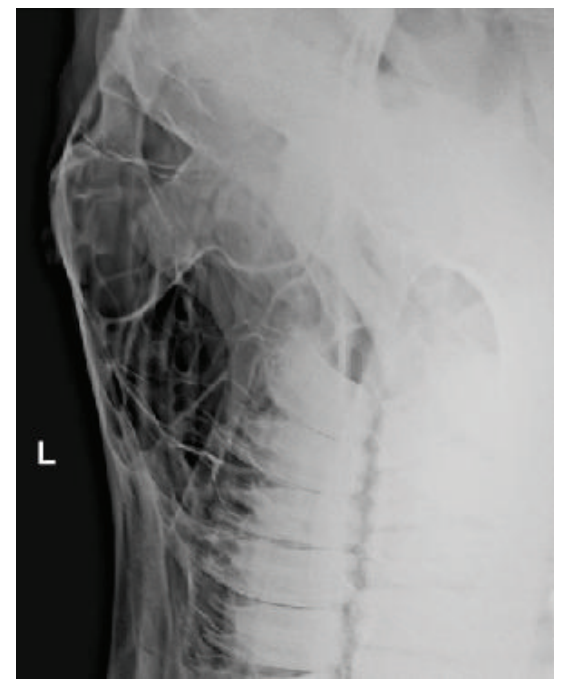

(c)

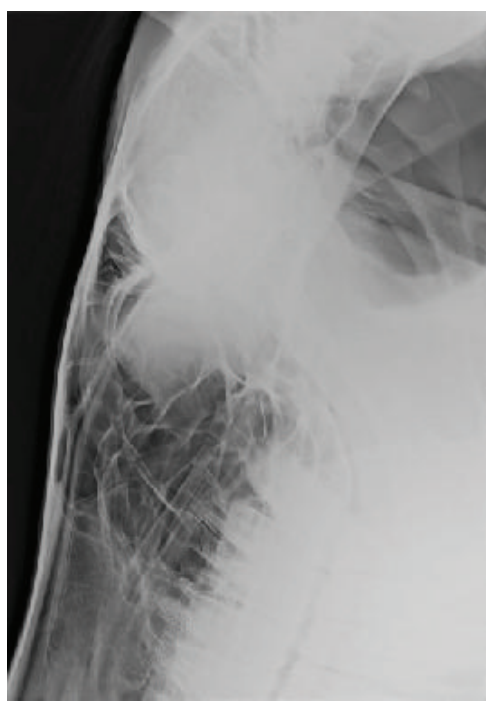

(b)

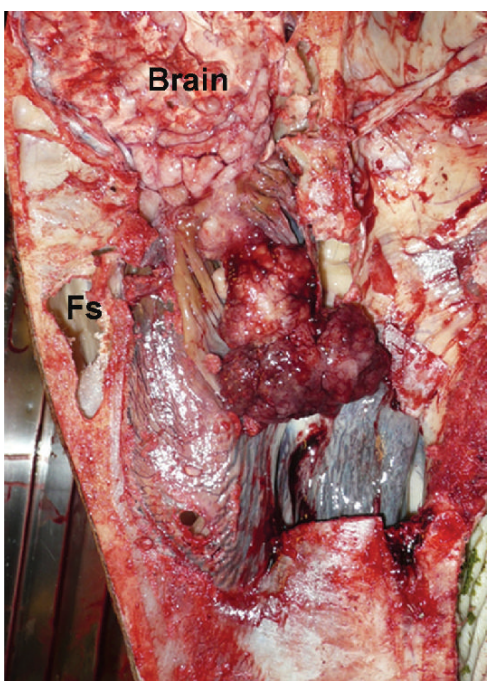

(d)

Figure 2: Radiographs of the skull at the time of second presentation in (a) dorsoventral and (b) lateral views. An indistinct soft tissue attenuating area is summating with the left turbinate region rostral to the rostral border of the choanae. (c) Oblique radiographic view of the left frontal and maxillary area. An indistinct soft tissue attenuating area is noted summating with the left lateral aspect of the ethmoid area. (d) Photographic image of the skull cut open in a left parasagittal plane. A dark red to tan mass is noted in the left ethmoid region attached to the caudal aspect of the turbinates. The mass does not extend through the cribriform plate. Fs: frontal sinus.

carcinoma, and undifferentiated carcinoma in one case each. Of the 7 horses, including the horse described in this case report, 2 had enlargement of local lymph nodes, one due to lymphoma and in the other there was no evidence of neoplastic cells. The horse described in this case report was the only one with confirmed distant metastasis of a sinonasal neoplasm.

\section{Discussion}

The incidence of sinonasal tumors in the population of equine patients seen at our facility is low and similar to that previously reported $[1,13,14]$. The horse diagnosed with nasal adenocarcinoma with metastasis to lung, liver, and distal humerus was unique as the first presenting complaint was related to the metastatic lesion and not the primary disease. Epistaxis from the nasal adenocarcinoma occurred later in the disease process and was not confirmed to be associated with the ongoing lameness until necropsy.

Neoplasia of the paranasal sinuses and nasal cavity is a relatively rare cause of epistaxis in horses. More common causes include exercise induced pulmonary hemorrhage, guttural pouch mycosis, ethmoid hematoma, and trauma [15]. Often the first diagnostic step in horses with suspected sinonasal disease is endoscopy. If further information regarding the lesion is desired, radiography is traditionally used. Radiographs have proven useful for initial evaluation in horses with sinonasal neoplasia; however they are limited 


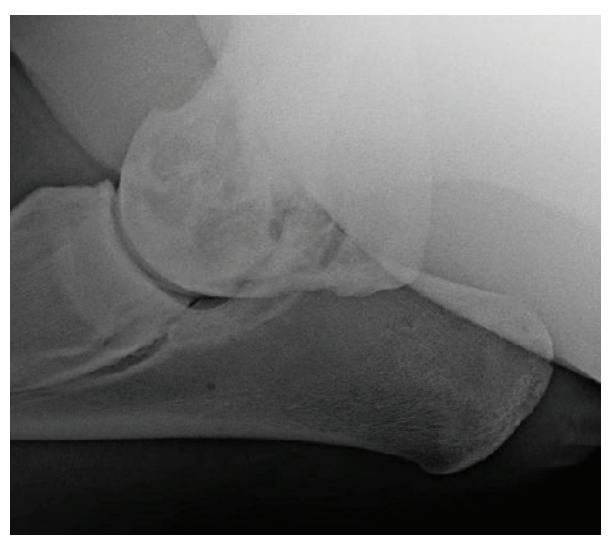

(a)

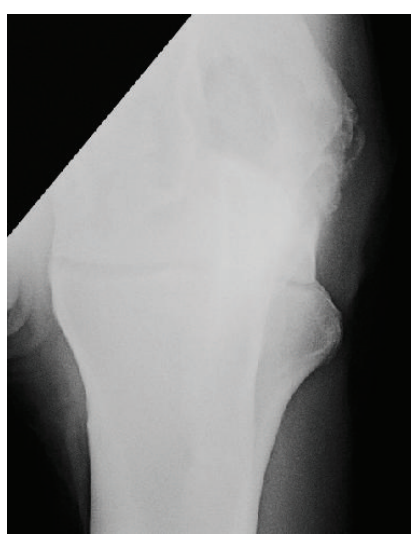

(b)

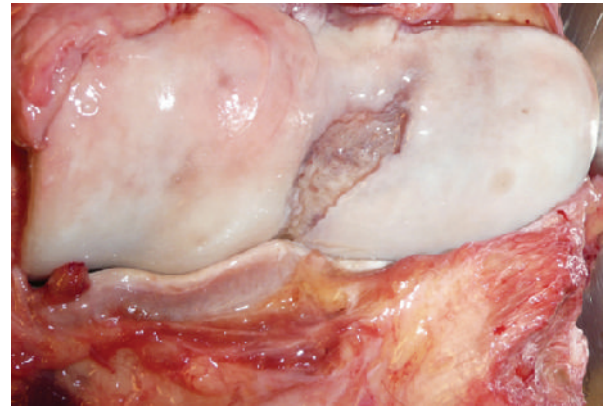

(c)

FIGURE 3: Radiographs of the right elbow at second presentation in (a) lateromedial and (b) craniocaudal view. Severe osteolysis of the right humeral condyle and marked irregularity and osteolysis of the lateral epicondyle of the right humerus are noted. (c) Photographic image of the right humeral condyle from the cranial aspect of the right humerus. A large defect secondary to osseous metastasis is noted in the centrolateral aspect of the condyle of the right humerus.

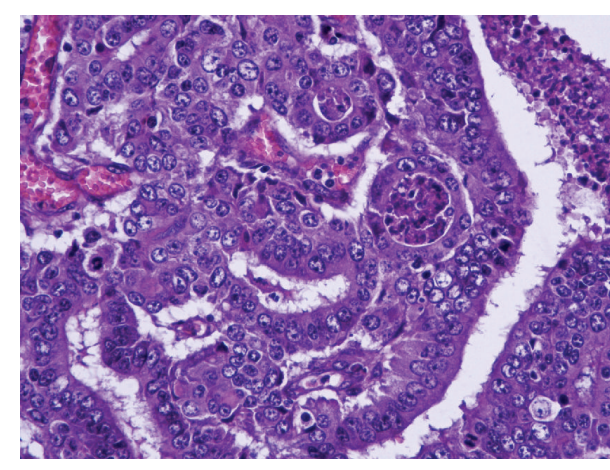

(a)

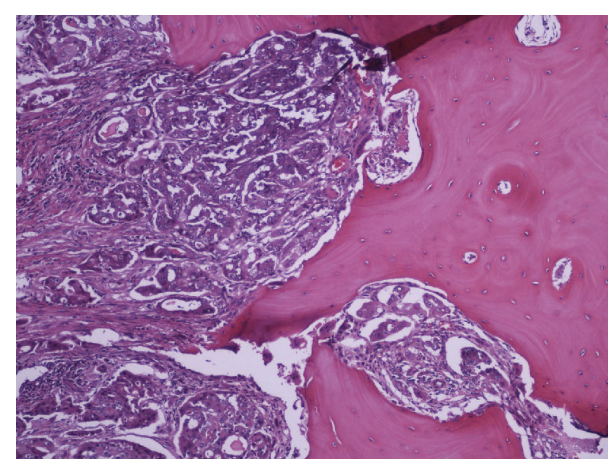

(b)

FIGURE 4: (a) Left ethmoid mass. The nasal adenocarcinoma effaces normal turbinates. The patterns of neoplastic cell distribution varied between tubular (shown) and solid. Hematoxylin and eosin, 400x. (b) Right humerus. Metastatic nasal adenocarcinoma, with tubular pattern, invading cortical bone. Hematoxylin and eosin, 100x.

in their ability to localize the lesion to the correct location. In this case radiography was the only imaging modality done, as treatment was not pursued. However, in cases of sinonasal neoplasia where surgery is warranted or further prognostic information is desired CT and MRI have shown to be beneficial $[16,17]$. The advantages of CT include the ability to better evaluate disease extension, assess involvement of surrounding osseous structures, and more precisely localize the disease [16]. MRI has been suggested to be more accurate than CT to determine the degree of brain involvement as well as damage to the soft tissues surrounding the orbit. A potential drawback of MRI is the intense sensitivity to inflammatory changes, which could lead to over diagnosis of a benign lesion [17].

Based on the presence of a nasal adenocarcinoma in this patient, the absence of other primary sites of adenocarcinoma 
in this horse, and metastatic epithelial cells invading the humerus, we present this as the first report of an adenocarcinoma of nasal or paranasal sinus origin metastasizing to bone in a horse. There have been reports in horses of adenocarcinomas originating from other sites that have metastasized to bone, causing significant lameness. This includes two cases of intestinal and one of renal adenocarcinoma with metastatic osseous involvement $[2,10,18]$. In all of the cases of metastatic adenocarcinoma to bone, the horses developed a significant lameness at some point in the disease process. This emphasizes the importance of evaluating the patient thoroughly as some clinical signs associated with the primary disease process may easily be overlooked.

This case report displays that sinonasal neoplasia in the horse can lead to distant metastatic disease. Clinical signs may be associated with the metastatic lesions and not necessarily be the result of the primary tumor. This case also serves as a reminder that osseous neoplasia is uncommon in horses but might be a significant source of lameness. Although metastatic disease associated with nasal adenocarcinoma in the horse is rare further evaluation for presence of metastatic disease should be performed prior to initiation of therapy.

\section{Conflict of Interests}

The authors declare that there is no conflict of interests regarding the publication of this paper.

\section{References}

[1] K. W. Head and P. M. Dixon, "Equine nasal and paranasal sinus tumours. Part 1: review of the literature and tumour classification," Veterinary Journal, vol. 157, no. 3, pp. 261-278, 1999.

[2] M. Oosterlinck, E. Raes, S. Verbraecken et al., "Severe lameness caused by metastatic renal adenocarcinoma of the third phalanx in a Warmblood mare," Equine Veterinary Education, vol. 23, no. 10, pp. 512-516, 2011.

[3] G. H. Edington and D. F. Cappell, "Adenocarcinoma of undescended testicle in the horse: (?) secondary growths in lung and mediastinum," Proceedings of the Royal Society of Medicine, vol. 24, pp. 1139-1140, 1931.

[4] F. L. Matheis, K. Birkmann, M. Ruetten, S. A. Pot, and B. M. Spiess, "Ocular manifestations of a metastatic adenocarcinoma in a horse," Veterinary Ophthalmology, vol. 16, no. 3, pp. 214-218, 2013.

[5] F. E. T. Pauwels, S. J. Wigley, J. S. Munday, and W. D. Roe, "Bilateral ovarian adenocarcinoma in a mare causing haemoperitoneum and colic," New Zealand Veterinary Journal, vol. 60, no. 3, pp. 198-202, 2012.

[6] G. P. Reppas, S. A. McClintock, P. J. Canfield, and G. F. Watson, "Papillary ductal adenocarcinoma in the mammary glands of two horses," Veterinary Record, vol. 138, pp. 518-519, 1996.

[7] J. A. Wright and G. B. Edwards, "Adenocarcinoma of the intestine in a horse: an unusual occurrence," Equine Veterinary Journal, vol. 16, no. 2, pp. 136-137, 1984.

[8] J. L. Davis, B. C. Gilger, K. Spaulding, I. D. Robertson, and S. L. Jones, "Nasal adenocarcinoma with diffuse metastases involving the orbit, cerebrum, and multiple cranial nerves in a horse,"
Journal of the American Veterinary Medical Association, vol. 221, no. 10, pp. 1460-1463, 2002.

[9] J. F. Zaruby, M. A. Livesey, and D. H. Percy, "Ethmoid adenocarcinoma perforating the cribriform plate in the horse," The Cornell Veterinarian, vol. 83, no. 4, pp. 283-289, 1993.

[10] L. M. East, P. F. Steyn, C. E. Dickinson, and A. A. Frank, “Occult osseous metastasis of a colonic adenocarcinoma visualized with technetium tc $99 \mathrm{~m}$ hydroxymethylene diphosphate scintigraphy in a horse," Journal of the American Veterinary Medical Association, vol. 213, pp. 1167-1170, 1998.

[11] C. M. Kane, P. Hoskin, and M. I. Bennett, "Cancer induced bone pain," British Medical Journal, vol. 350, article h315, 2015.

[12] J. M. Bush, R. L. Fredrickson, and E. J. Ehrhart, "Equine osteosarcoma: a series of 8 cases," Veterinary Pathology, vol. 44, no. 2, pp. 247-249, 2007.

[13] C. H. Boulton, "Equine nasal cavity and paranasal sinus disease: a review of 85 cases," Journal of Equine Veterinary Science, vol. 5, pp. 267-275, 1985.

[14] P. M. Dixon and K. W. Head, "Equine nasal and paranasal sinus tumours: part 2: a contribution of 28 case reports," Veterinary Journal, vol. 157, no. 3, pp. 279-294, 1999.

[15] B. P. Smith, Large Animal Internal Medicine, Elsevier Health Sciences, 4th edition, 2008.

[16] D. D. Cissell, E. R. Wisner, J. Textor, F. C. Mohr, P. V. Scrivani, and A. P. Théon, "Computed tomographic appearance of equine sinonasal neoplasia," Veterinary Radiology and Ultrasound, vol. 53, no. 3, pp. 245-251, 2012.

[17] C. Tessier, A. Brühschwein, J. Lang et al., "Magnetic resonance imaging features of sinonasal disorders in horses," Veterinary Radiology and Ultrasound, vol. 54, no. 1, pp. 54-60, 2013.

[18] H. W. Jann, M. A. Breshears, R. W. Allison et al., "Occult metastatic intestinal adenocarcinoma resulting in pathological fracture of the proximal humerus," Equine Veterinary Journal, vol. 41, no. 9, pp. 915-917, 2009. 

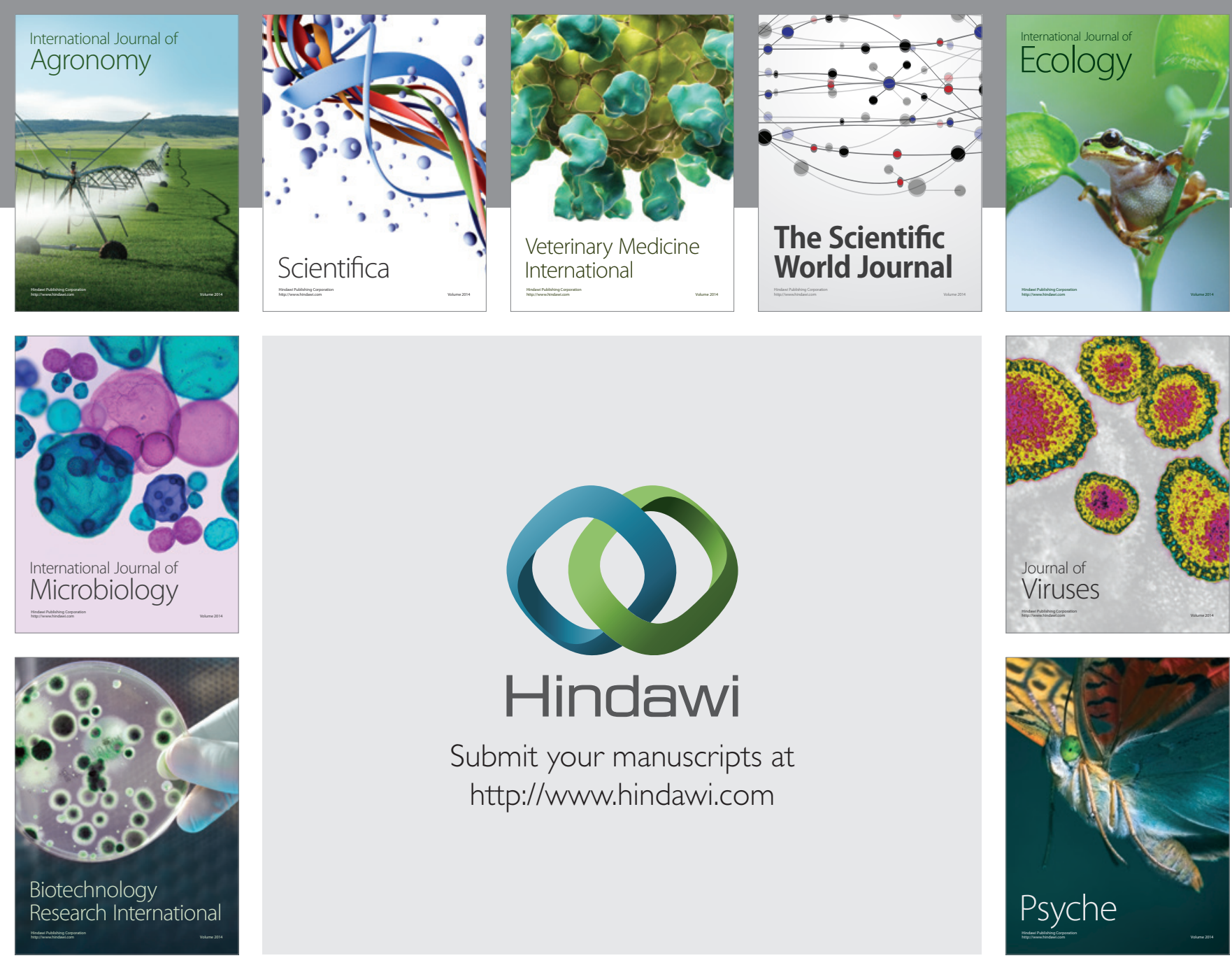

Submit your manuscripts at http://www.hindawi.com
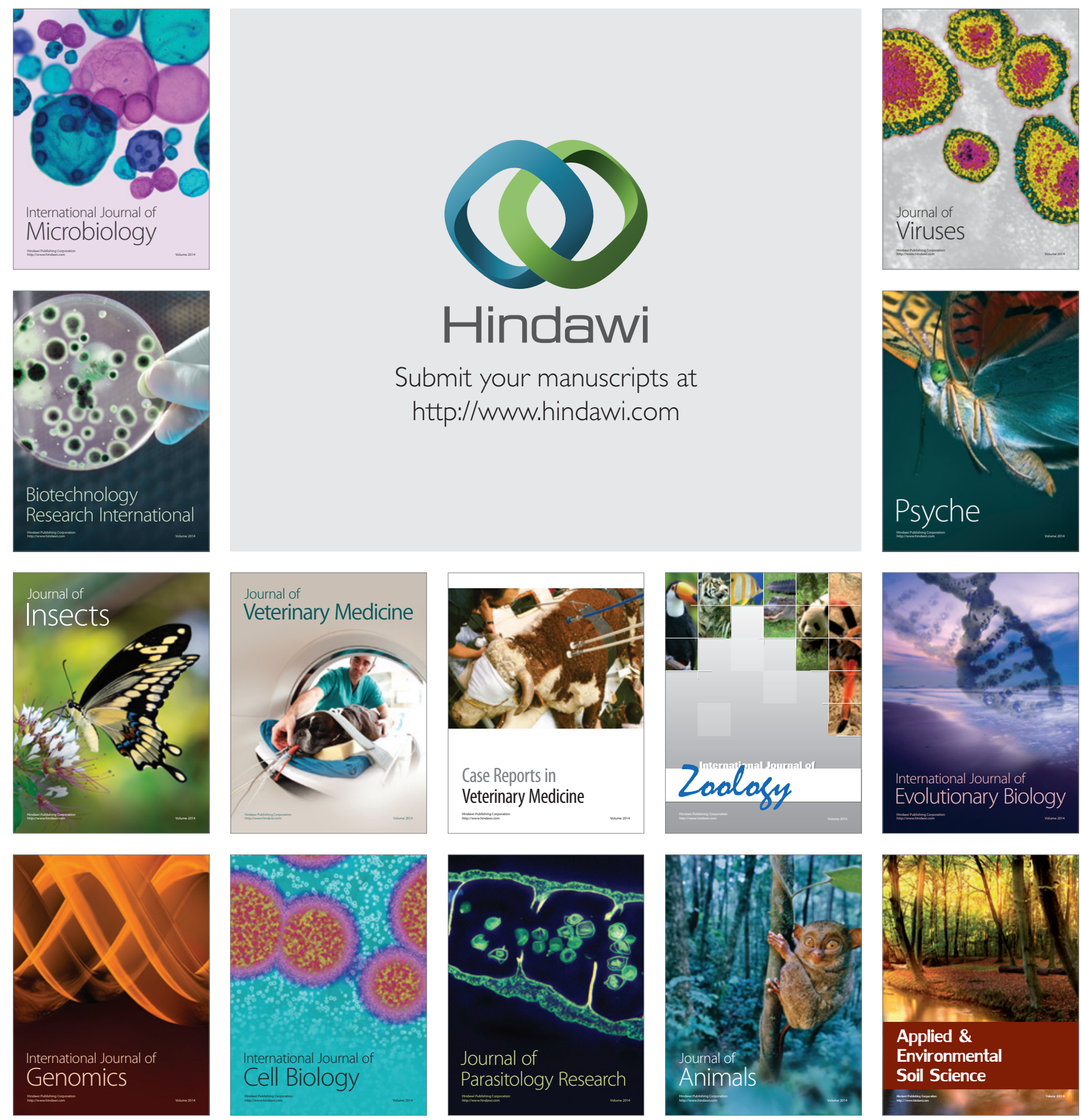